\title{
Rotation Invariant Texture Classification using Fuzzy Logic
}

\author{
Dattatraya S. Bormane \\ Department of Electronics and \\ Telecommunication \\ Engineering, JSPM's Rajarshi \\ Shahu College of Engineering, \\ Pune, Maharashtra State, \\ India.
}

\author{
Shailendrakumar M. \\ Mukane \\ Department of Electronics and \\ Telecommunication \\ Engineering, SVERI's College \\ of Engineering, Pandharpur, \\ Maharashtra State, India.
}

\author{
Sachin R. Gengaje \\ Department of Electronics \\ Engineering, Walchand \\ Institute of Technology, \\ Solapur, Maharashtra State, \\ India.
}

\begin{abstract}
In this paper, we develop a scale invariant texture classification method based on Fuzzy logic. It is applied for the classification of texture images. Texture is a common property of any surface having uncertainty. Two types of texture features are extracted one using Discrete Wavelet Transform (DWT) and other using Co-occurrence matrix. Cooccurrence features are obtained using DWT coefficients. Two features are obtained from each sub-band of DWT coefficients upto fifth level of decomposition and eight features are extracted from co-occurrence matrix of whole image and each sub-band of first level DWT decomposition. The fuzzy classification is achieved in two steps, fuzzification step, and rule generation step. The performance is measured in terms of Success Rate. This study showed that the proposed method offers excellent scale invariant texture classification Success Rate. Also wavelet features like standard deviation, combination of energy and standard deviation along with some proposed hybrid feature sets outperform the other feature sets. This success rate is comparatively high when compared with results published earlier.
\end{abstract}

\section{Keywords}

Texture Classification, Rotation Invariance, Fuzzy Logic, Discrete wavelet Transform.

\section{INTRODUCTION}

Content Based Image Retrieval (CBIR) method where in images are expressed by their visual content of images. The key component of any CBIR system is classification of images. Texture, Shape, and Colour are the general visual content features of image. Texture features are very important because it is an intrinsic property of virtually all surfaces such as skin, bricks, tree, fabric, grass, hair, clouds, etc. It contains information about the structural arrangement of surfaces and their relationship to the surrounding environment [1]. Also textures can refer to the visual patterns that have properties of homogeneity that will result from the presence of colour and intensity. Texture analysis is basic step to many applications such as industrial monitoring of product quality, remote sensing of earth resources, medical diagnosis, image retrieval systems, etc.

Weszka et al. [2] compared the classification performance of Fourier power spectrum, second order Gray Level Cooccurrence Matrix (GLCM), and first order statistics of gray level differences for terrain samples and commented that Fourier methods performed poorly. Haralick [1] suggested GLCM texture features and used these features to analyze remotely sensed images. Wan et al. [3] presented comparative study of four texture analysis methods such as gray level Runlength method[RLM], Co-occurrence matrix method, Histogram method, and Auto-correlation method and shown that Co-occurrence method is superior. Wavelet Transform [4, 5] provides a multi-resolution approach for the problem at hand. Smith and Chang [6] used mean and variance extracted from wavelet sub-band coefficients, as the texture representation.

Classification methods can be divided into categories such as parametric, non-parametric, stochastic methods, non-metric methods [7]. Classification task involves classifying images based on the feature vectors provided by the feature extraction methods. If no prior parameterized knowledge about the probability structure then classification is based on nonparametric techniques. That classification will be based on information provided by training samples alone. These techniques include fuzzy classification, neural network approach, etc. Engin Avci [8] used multilayer perceptron neural network classifier to classify selected texture images. Turkoglu and Avci [9] presented a comparison of wavelet support vector machine (W-SVM) and wavelet-adaptive network based fuzzy inference system (W-ANFIS) approaches for texture image classification. Both W-SVM and W-ANFIS methods are used for classification of the 22 texture images. G. Schaefer et al. [10] used fuzzy classification for thermograph based breast cancer analysis using statistical features. Mukane et al. carried out the scale invariance [11], size invariance [12], and rotation invariance [13] with wavelet and co-occurrence matrix based features using fuzzy logic classifier. Laine and Fan [14] uses standard wavelet packet energy signature for texture classification. Pun and Lee [15] had Log-polar Wavelet Signature with Mahalanobis Classifier for Scale and Rotation invariant texture classification. Cui et al. [16] perform experiment for Rotation invariant texture classification based on Radon transform and multi-scale analysis with Mahalanobis classifier. Hiremath and Shivshankar [17] Wavelet based cooccurrence histogram features for texture classification with k-NN classifier. Arivazhagan et al. [18] used Gabor features for rotation invariant texture classification with minimum distance classifier.

In this study, rotation invariant texture classification method is proposed using fuzzy logic. 25 texture images are taken from the Brodatz texture Album. Fifteen feature sets are formed from the extracted features of DWT based features, and DWT-GLCM based feature set. The average success rate of fuzzy classifier to classify texture images with training to 
testing ratio $1: 1$ is obtained. These results are compared with some of the best results available in the literature.

\section{FEATURE EXTRACTION}

Features of texture images are extracted as a first step in the classification and retrieval application. Pyramid structured DWT is used to decompose the textures up to five levels. Wavelet Statistical Features (WSF) is obtained [11]. Gray Level Co-occurrence matrix (GLCM) is derived for distance vector $d(i, j)$ i.e. offset is taken as $d(0,1)$. This matrix is obtained for whole image and four sub-bands of first level DWT of the image. From the GLCM the co-occurrence parameters namely contrast, inverse difference moment, energy, norm entropy, local homogeneity, cluster shade, cluster prominence, \& maximum probability are obtained[9, 15]. These features are called as Wavelet Co-occurrence Features (WCF).

\section{CLASSIFICATION}

A fuzzy logic system is a nonlinear mapping of an input feature vector into a scalar output [19]. Fuzzy logic provides innovative tools to handle the complex and ill-defined systems where classical tools become unsuccessful. Fuzzy systems are universal approximators of non linear functions. Two aspects are important in fuzzy system one generating the best rule set and second the membership function. These should relate properly the independent and dependent variable.

Membership function maps elements of a given universal set which is always a crisp set in to real numbers in a specific range. The most commonly used membership functions $\mu(x)$, are triangular, trapezoidal, piecewise linear, or Gaussian. Whether a particular membership function is suitable or not can be determined only in the context of a particular application. The membership function required for the fuzzy classifier in the present research is Gaussian function. Inputs to the fuzzy system are WSF and WCF features. The outputs of the system are specific texture image. Fuzzy sets with Gaussian membership functions are used to define these input variables. These fuzzy sets can be defined using the following equation [20].

$$
\boldsymbol{\mu}(\boldsymbol{f})=e^{-0.5\left(\frac{f-m}{\sigma}\right)^{2}}
$$

where $\mathrm{m}$ is the mean of the fuzzy set and $\sigma$ is the standard deviation from the mean.

\section{RESULTS AND DISCUSSION}

In this experiment twenty five texture images [9] from the Brodatz texture [21] are used for classification. Texture images are then sampled to $256 \times 256$ sizes [13]. To have different Rotation, 3000 texture samples with $0^{\circ}, 30^{\circ}, 60^{\circ}, 120^{\circ}$ rotation each is taken and mixed. This leads to 12000 samples. This way 12000 samples used for training and testing of Fuzzy logic Classifier is carried for another 12000 samples generated in the very same way. Ratio of training to testing is maintained $1: 1$. In this experiment pyramid structured type of DWT is used with $\mathrm{dB} 2$ as a wavelet filter [16].

Feature database is created using wavelet decomposed subbands up to fifth level of decomposition. Total number of subbands up to fifth level will be 20. Energy and standard deviations of each sub-band coefficients are calculated. These features are stored as WSF. Another feature database is obtained using eight co-occurrence features by finding the cooccurrence matrix of original sample image and 4- sub-bands of the 1-level DWT coefficients' co-occurrence matrix. These are stored as WCF. This way, maximum WSF will be
$20 \times 2=40$ for five level decomposition and maximum WCF will be $5 \times 8=40$ for a sample. Feature sets Classification is carried out for following feature sets.

1) WSF1- Energy only.

2) WSF2- Standard Deviation

3) WSF3- Energy + Standard Deviation

4) WCF1- proposed Five Co-occurrence features viz. contrast, energy, local homogeneity, cluster shade, and cluster prominence.

5) WCF2- Eight Co-occurrence features viz. contrast, inverse difference moment, energy, norm entropy, local homogeneity, cluster shade, cluster prominence, \& maximum probability.

6) WCF3- proposed Four Co-occurrence features viz. contrast, inverse difference moment, norm entropy, energy.

7) HWSCF1- proposed WSF1+WCF1

8) HWSCF2- proposed WSF2+WCF1

9) HWSCF3- proposed WSF3+WCF1

10) HWSCF4- WSF1+WCF2

11) HWSCF5- WSF2+WCF2

12) HWSCF6- WSF3+WCF2

13) HWSCF7- proposed WSF1+WCF3

14) HWSCF8- proposed WSF2+WCF3

15) HWSCF9- proposed WSF3+WCF3

Performance of the above feature sets is tested with the help of a fuzzy classifier in terms of Success rate. Let $\mathrm{N}_{\mathrm{T}}$ be the no. of samples to be tested and out of that if the system correctly classifies $\mathrm{N}_{\mathrm{C}}$ times then success rate of the system for the fuzzy rule as a percentage is given by

$$
S_{R}=\frac{N_{C}}{N_{T}} x 100
$$

Fig.1 shows graph of average success rate versus number of features for various feature sets. By observation of Fig. 1(a), (b) and (c) it is noticed that WSF3, proposed HWSCF7, and HWSCF5 offers good Classification performance but not consistent. The proposed feature sets HWSCF2, HWSCF3, HWSCF8, HWSCF9, WSF2, and HWSCF6 offers 100\% success rate for Rotation Invariance.

The results of this research work are compared with some of the earlier work published in the literature as presented in the Table 1. 


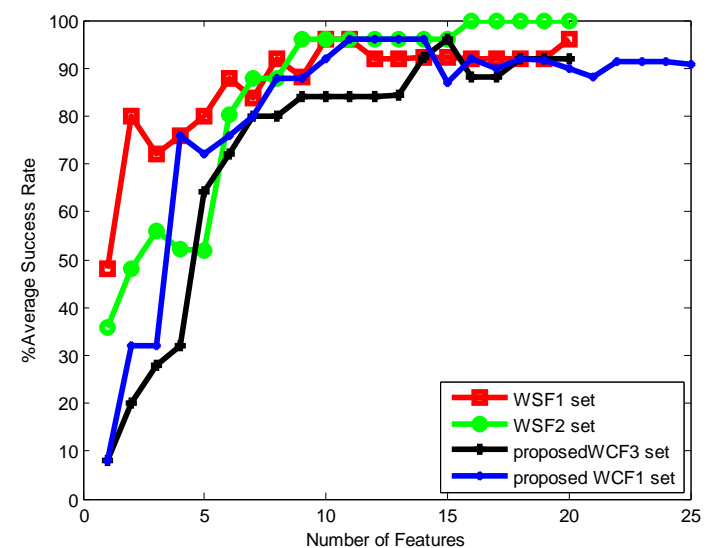

(a)

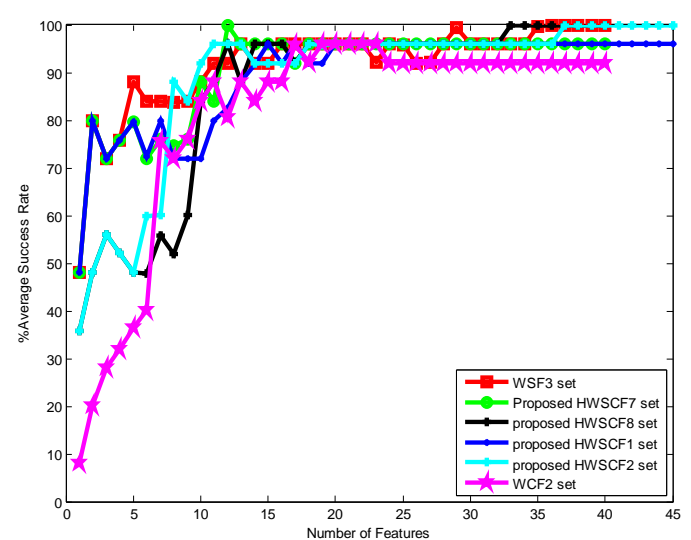

(b)

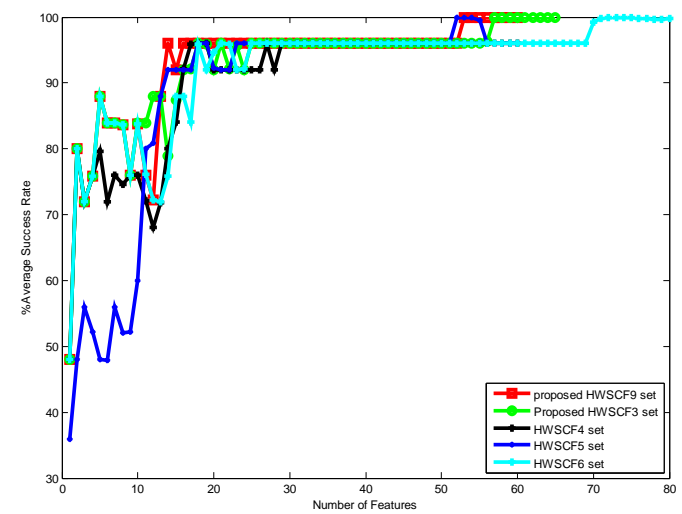

(c)

Fig 1: (a)-(c) Performance of Fuzzy Classifier for Rotation Invariance of Textures with various Feature Sets for 12000 samples of $256 \times 256$ sizes
Table 1. Comparison of Proposed method results with Earlier published methods

\begin{tabular}{|l|c|c|}
\hline $\begin{array}{l}\text { Texture Classification } \\
\text { Methods from } \\
\text { Literature }\end{array}$ & $\begin{array}{l}\text { \% Correct } \\
\text { Classification } \\
\text { Rate }\end{array}$ & $\begin{array}{c}\text { Number of } \\
\text { Features }\end{array}$ \\
\hline $\begin{array}{l}\text { Gabor wavelets based } \\
\text { rotation invariant features } \\
\text { with minimum distance } \\
\text { classifier(Arivazhagan et } \\
\text { al. 2006)[18] }\end{array}$ & 95.60 & 60 \\
\hline $\begin{array}{l}\text { Radon Transform features } \\
\text { with Mahalanobis } \\
\text { Classifier (Cui et al. }\end{array}$ & 92.2 & 21 \\
2006)[16] & & \\
\hline $\begin{array}{l}\text { Gabor Features with K-NN } \\
\text { classifier (Hiremath and } \\
\text { Shivshankar 2008)[17] }\end{array}$ & 96.84 & 384 \\
\hline $\begin{array}{l}\text { Proposed DWT and } \\
\text { GLCM based Fuzzy logic } \\
\text { Classifier system }\end{array}$ & 100 & 12 \\
\hline
\end{tabular}

\section{CONCLUSION}

In this study, traditional feature sets as well as proposed feature sets are tested with fuzzy classifier to classify rotation invariant texture images. The some proposed feature sets as well as traditional feature sets outperform other feature sets. Fuzzy logic classifier tested for large sample size of 12000 providing excellent results. The proposed hybrid feature set HWSCF2, HWSCF3, HWSCF8, and HWSCF9 offers 100\% success rate for rotation invariant texture classification. Fuzzy logic classifier performs excellently with training to testing ratio of $1: 1$ and consistency of excellent success rate. The results are compared with existing literature results which shows that our method outperform the others.

\section{REFERENCES}

[1] R. M. Haralick, K. Shanmugam, and I. Dinstein (1973) Textural features for image classification, IEEE Trans. on Systems, Man, and Cybernetics, 3, 610-621.

[2] J. Weszka, C. Dyer, and A. Rosenfeld (1976) A comparative study of texture measures for terrain classification, IEEE Trans. on Systems, Man, and Cybernetics, 6(4).

[3] Y. Wan, J. Du, D. Huang, Z. Chi, Y. Cheung, X. Wang, G. Zhang (2004) Bark Texture Feature Extraction Based on Statistical Texture Analysis, In Proceedings of 2004 Int. Sympo. On Intelligent multimedia, Video \& Speech processing, Hong Kong.

[4] I. Daubechies (1990) The wavelet transform, timefrequency localization and signal analysis, IEEE Trans. on Information Theory, 36, 961-1005.

[5] S. G. Mallat (1989) A theory for multi-resolution signal decomposition: the wavelet representation”, IEEE Trans. on PAMI, 11, 674-693.

[6] J. R. Smith and S. F. Chang (1994) Transform features for texture classification and discrimination in large image databases, In Proceedings of IEEE International Conference on Image Processing.

[7] R.O. Duda, P.E. Hart, D.G. Stork (2006) Pattern Classification, John Wiley, and Sons. 
[8] E.Avci (2007) An expert system based on Wavelet Neural Network-Adaptive Norm Entropy for scale invariant texture classification, Journal on Experts Systems with Applications, 32, 919-926.

[9] I. Turkoglu and E.Avci (2008) Comparison of waveletSVM and Wavelet-adaptive network based fuzzy inference system for texture classification, Journal on Digital Signal Processing, 18, 15-24.

[10] G. Schaefer, M. Zavisek, T.Nakashima (2009) Thermography based breast cancer analysis using statistical features and fuzzy classification, Journal of Pattern Recognition, 47, 1133-1137.

[11] S.M. Mukane, S.R. Gengaje, and D.S. Bormane (2011) On Scale Invariance Texture Image Retrieval using Fuzzy Logic and Wavelet Co-occurrence based Features, International Journal of Computer Applications, 18(3), 10-17.

[12] S.M. Mukane, D.S. Bormane, and S.R. Gengaje (2011) On Size Invariance Texture Image Retrieval using Fuzzy Logic and Wavelet based Features, International Journal of Applied Engineering Research, 6(6), 1297-1310.

[13] S. M. Mukane, D. S. Bormane and S. R. Gengaje (2011) Wavelet and Co-occurrence Matrix based Rotation Invariant Features for Texture Image Retrieval using Fuzzy Logic, International Journal of Computer Applications, 24(7), 1-5.
[14] Laine A. and Fan J. (1993) Texture classification by wavelet packet signatures IEEE transactions on PAMI. 15(11), 1186-1191.

[15] Pun C. and Lee, M. (2003) Log-polar wavelet energy signatures for rotation and scale invariant texture classification. IEEE Transactions on PAMI, 25(5), 590603.

[16] Cui, P., Li, J., Pan, Q., Zhang, H. (2006), Rotation and scaling invariant texture classification based on Radon transform and multi-scale analysis, Pattern Recognition Letters, 27, 408-413.

[17] Hiremath P.S. Shivshankar S. (2008) Wavelet based cooccurrence histogram features for texture classification with an application to script identification in a document image, Pattern Recognition Letters, 29, 1182-1189.

[18]S. Arivazhagan, L. Ganesan, S. Padam Priyal (2006) Texture classification using Gabor wavelets based rotation invariant features, Pattern Recognition Letters 27, 1976-1982.

[19] Kosko, B.(1997) Fuzzy Engineering, Prentice Hall, New Jersey.

[20] P.M. Pawar and R. Ganguli (2003) Genetic fuzzy system for damage detection in beams and helicopter rotor blades, Journal of Computer methods in applied mechanics and engineering, 192, 2031-2057.

[21] P. Brodatz (1966) Textures: A Photographic Album for Artists and Designers, Dover, New York. 